NBER WORKING PAPER SERIES

\title{
THE CORONAVIRUS EPIDEMIC CURVE IS ALREADY FLATTENING IN NEW YORK CITY
}

\author{
Jeffrey E. Harris \\ Working Paper 26917 \\ http://www.nber.org/papers/w26917
NATIONAL BUREAU OF ECONOMIC RESEARCH
1050 Massachusetts Avenue
Cambridge, MA 02138
April 2020

The comments of the following individuals are greatly appreciated: Gil Brodsky, Peter Diamond, Denise Everett, Daniel Geselowitz, Ali Harris, Dena Harris, Kathryn Blackmond Laskey, Ken Laskey, Zoe Lazarre, Marylee Maendler, Melissa Oppenheim Margolis, Heide O'Connell, Brina Sedar, Susan Goldberg Simon, Rivana Cohen Stadtlander and Mark Weinstein. The opinions expressed here are solely those of the author and do not represent the views of the Massachusetts Institute of Technology, the National Bureau of Economic Research, or any other individual or organization. The author has received no direct or indirect remuneration for this article, and has no conflicts of interest to declare. The views expressed herein are those of the author and do not necessarily reflect the views of the National Bureau of Economic Research.

NBER working papers are circulated for discussion and comment purposes. They have not been peer-reviewed or been subject to the review by the NBER Board of Directors that accompanies official NBER publications.

(C) 2020 by Jeffrey E. Harris. All rights reserved. Short sections of text, not to exceed two paragraphs, may be quoted without explicit permission provided that full credit, including () notice, is given to the source. 
The Coronavirus Epidemic Curve is Already Flattening in New York City

Jeffrey E. Harris

NBER Working Paper No. 26917

April 2020

JEL No. I1,I12,I18,I28

\begin{abstract}
$\underline{\text { ABSTRACT }}$
New York City has been rightly characterized as the epicenter of the coronavirus pandemic in the United States. Just one month after the first cases of coronavirus infection were reported in the city, the burden of infected individuals with serious complications of COVID-19 has already outstripped the capacity of many of the city's hospitals. As in the case of most pandemics, scientists and public officials don't have complete, accurate, real-time data on the path of new infections. Despite these data inadequacies, there already appears to be sufficient evidence to conclude that the curve in New York City is indeed flattening. The purpose of this report is to set forth the evidence for - and against - this preliminary but potentially important conclusion. Having examined the evidence, we then inquire: if the curve is indeed flattening, do we know what caused to it to level off?
\end{abstract}

Jeffrey E. Harris

Department of Economics, E52-422

MIT

50 Memorial Drive

Cambridge, MA 02142

and NBER

jeffrey@mit.edu 


\section{Introduction}

New York City has been rightly characterized as the epicenter of the coronavirus pandemic in the United States. Just one month after the first cases of coronavirus infection were reported in the city, the burden of infected individuals with serious complications of COVID-19 has already outstripped the capacity of many of the city's hospitals (Harris and Sellers 2020). In response to the growing threat, Mayor de Blasio on March 16, 2020 issued an order limiting gatherings and closing numerous places of congregation. On March 21, Governor Andrew Cuomo issued a statewide order that all New Yorkers except those working in essential business had to stay home. The objective of these social distancing measures has been to slow down the transmission of infection - or "flatten the curve" of the epidemic - and thus relieve the burden on the city's healthcare system and buy time until effective treatments and possibly a vaccine can be developed.

As in the case of most pandemics, scientists and public officials don't have complete, accurate, real-time data on the path of new infections. In the case of the coronavirus epidemic, data interpretation is further complicated by the fact that about half of all infected individuals are asymptomatic (that is, they have few if any symptoms) and don't ordinarily seek medical attention. In the absence of mandatory, comprehensive testing, it is difficult to assess exactly where we are in the course of the epidemic and whether the curve is indeed flattening.

Despite these data inadequacies, there already appears to be sufficient evidence to conclude that the curve in New York City is indeed flattening. The purpose of this report is to set forth the evidence for - and against - this preliminary but potentially important conclusion. Having examined such evidence, we then inquire: if the curve is indeed flattening, do we know what caused to it to level off?

\section{Daily Counts of Newly Reported Infections}

Figure 1 shows the main evidence in favor of a flattening of the curve. Plotted are the numbers of new infections reported by the New York City Department of Health each day from March 1-30, 2020 (New York Department of Health and Mental Hygiene 2020b). The vertical axis gauges the numbers of infections on a logarithmic scale. The pink-colored data points cover the period from March 1-20, while the mango-colored points cover the period from March 21 onward. We prospectively chose the March 21 cutoff date to correspond to the effective date of Gov. Cuomo's order, before we observed the subsequent data points. A straight-line has been 
fitted to the data from March 1-20, based upon Poisson regression, as described further in Appendix 2. The slope of the straight line corresponds to an estimated doubling time of 1.3 days.

The mango-colored points in Figure 1 show a marked deviation from the exponential time-path projected from the pink-colored points through March 20. The number of reported new infections on March 30 was 4,603. If the path of the epidemic had continued at the pace predicted from the fitted line, new daily infections would by now exceed 100 thousand.

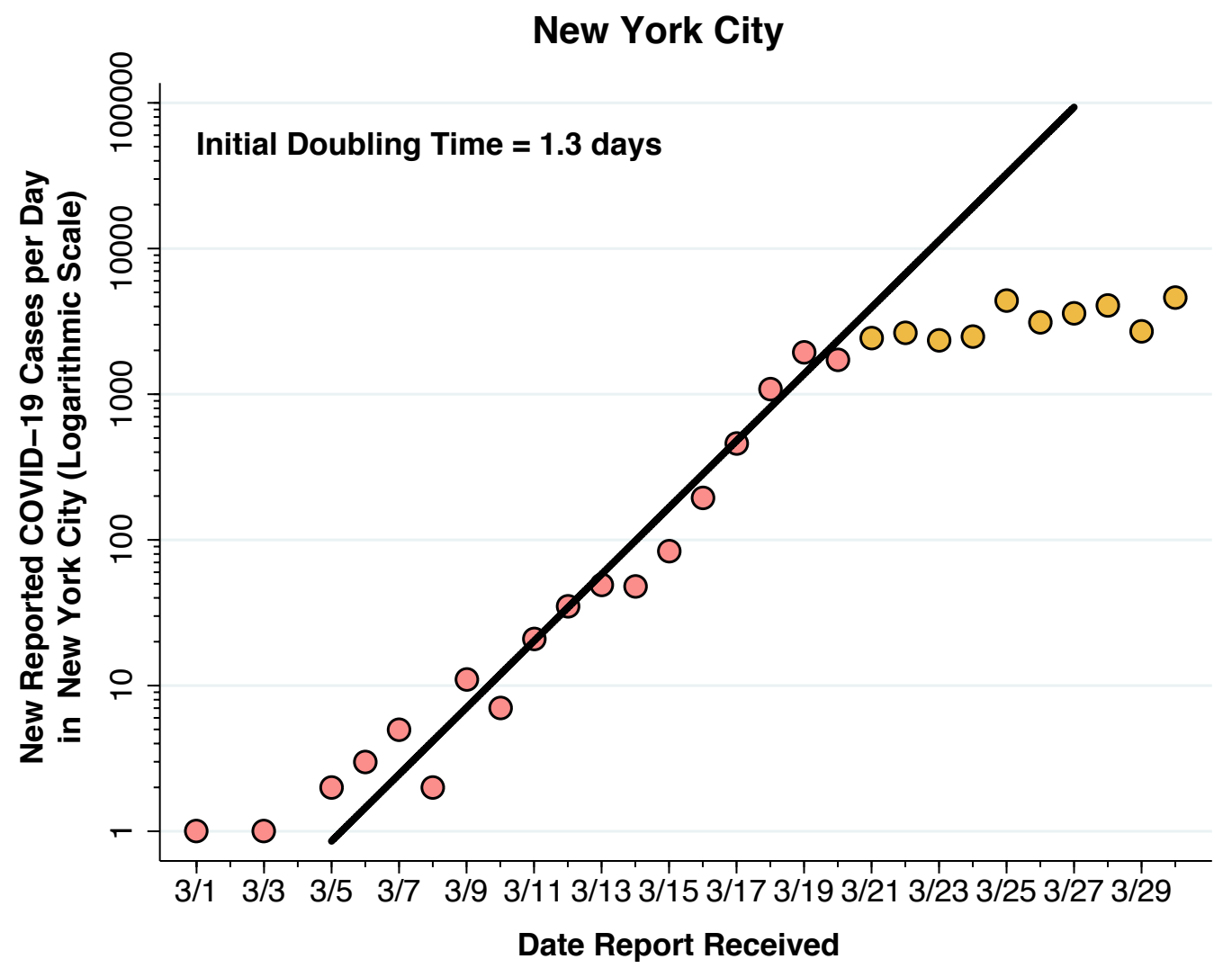

Figure 1. New Reported COVID-19 Cases per Day in New York City (Logarithmic Scale), Based on Date Reported. Best-fit trend line (Poisson regression) and estimated doubling time based upon observations through 3/20/2020, shown as pink data points. Observations from 3/21/2020 onward shown as mango data points.

As discussed in Appendix 1, the initial exponential takeoff in Figure 1 is exactly what the most widely used mathematical model would predict for an epidemic where virtually the entire population is susceptible. And the extremely short doubling time is exactly what mathematical modelers would expect in a population so incredibly dense as New York City. That's because the closer people are together, the higher is the likelihood that an infected person carrying the virus will make contact with a susceptible person. By way of comparison, Figure 2 shows the comparable plot for Los Angeles County (County of Los Angeles Public Health 2020), where we 
haven't thus far seen a definite flattening of the curve, but where the estimated doubling time through March 20 was 2.6 days. The population density of Los Angeles County is about 2,500 persons per square mile, in contrast to about 26,400 persons per square mile in New York City.

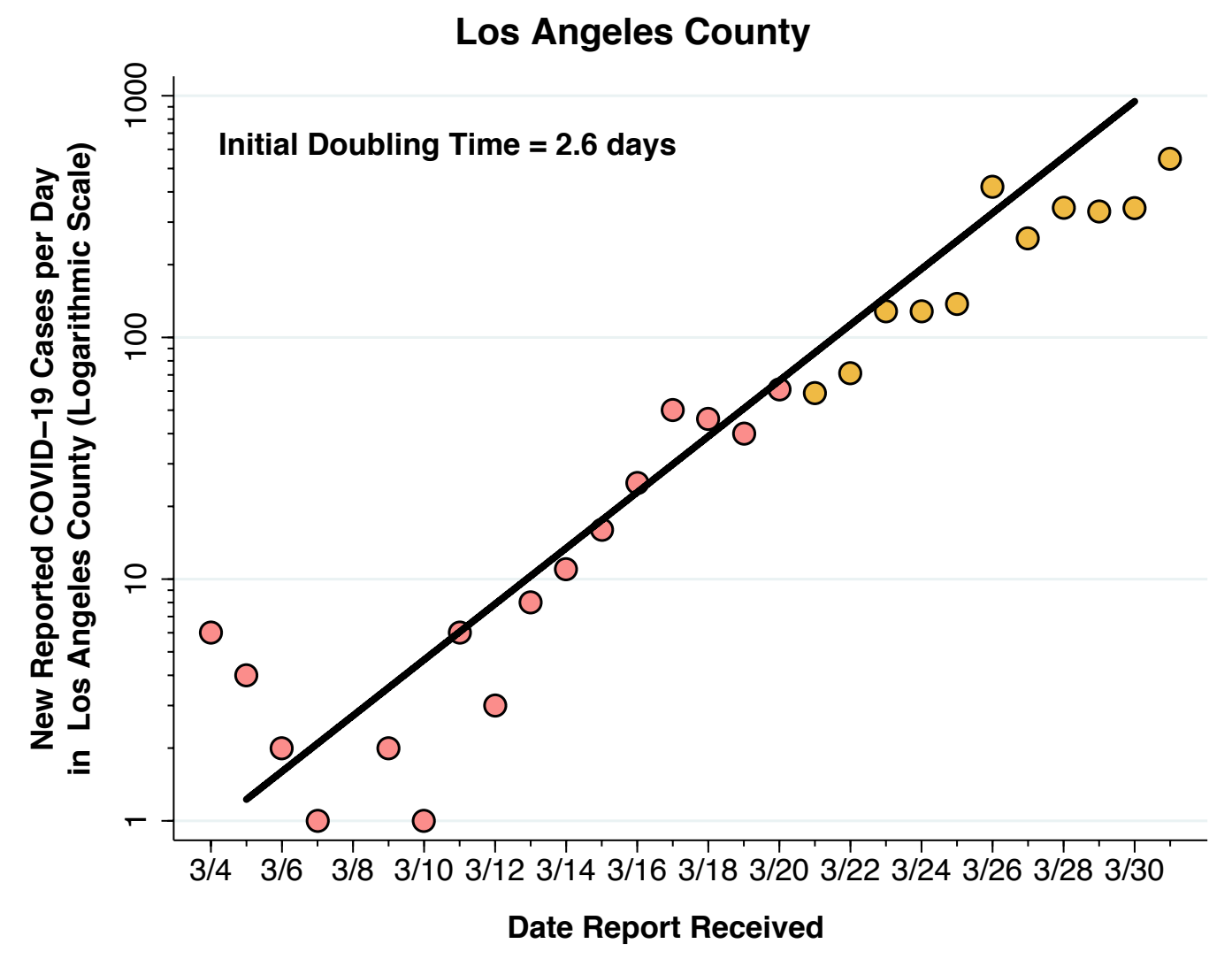

Figure 2. New Reported COVID-19 Cases per Day in Los Angeles County (Logarithmic Scale), Based on Date Reported. Best-fit trend line (Poisson regression) and estimated doubling time based upon observations through 3/20/2020, shown as pink data points. Observations from 3/21/2020 onward shown as mango data points.

\section{Alternative Explanation \#1. Supply Constraints on Testing}

If Figure 1 does not represent the actual path of new daily infections in New York City, then we need to come up with an alternative explanation. One possibility is that the apparent flattening of the mango data points is the result of supply constraints on testing. That is, the supply of tests - including the personal protective equipment required to perform those tests was simply inadequate to keep up with the demand. On March 20, in fact, the New York City Department of Public Health issued a memo observing, "Healthcare resources must be saved to treat the sickest patients who require inpatient and critical care." The Department directed providers and hospitals to "immediately stop testing non-hospitalized patients for COVID-19 unless test results will impact the clinical management of the patient. In addition, do not test 
asymptomatic people, including HCWs [healthcare workers] or first responders." (New York Department of Health and Mental Hygiene 2020a)

The fact that the Department of Health issued such a directive does not mean that it was fully complied with. As clinicians well know, the proviso "unless test results will impact clinical management" is just too squishy. Figure 3 addresses this question. The green-color data points along the top of Figure 3 show the numbers of tests performed in New York City on each day from March 8-27, 2020. The light gray-colored points show the numbers of such tests that were reported as positive, while the orange-colored points the numbers of such tests that were linked to a patient who was ultimately hospitalized (New York Department of Health and Mental Hygiene 2020d).

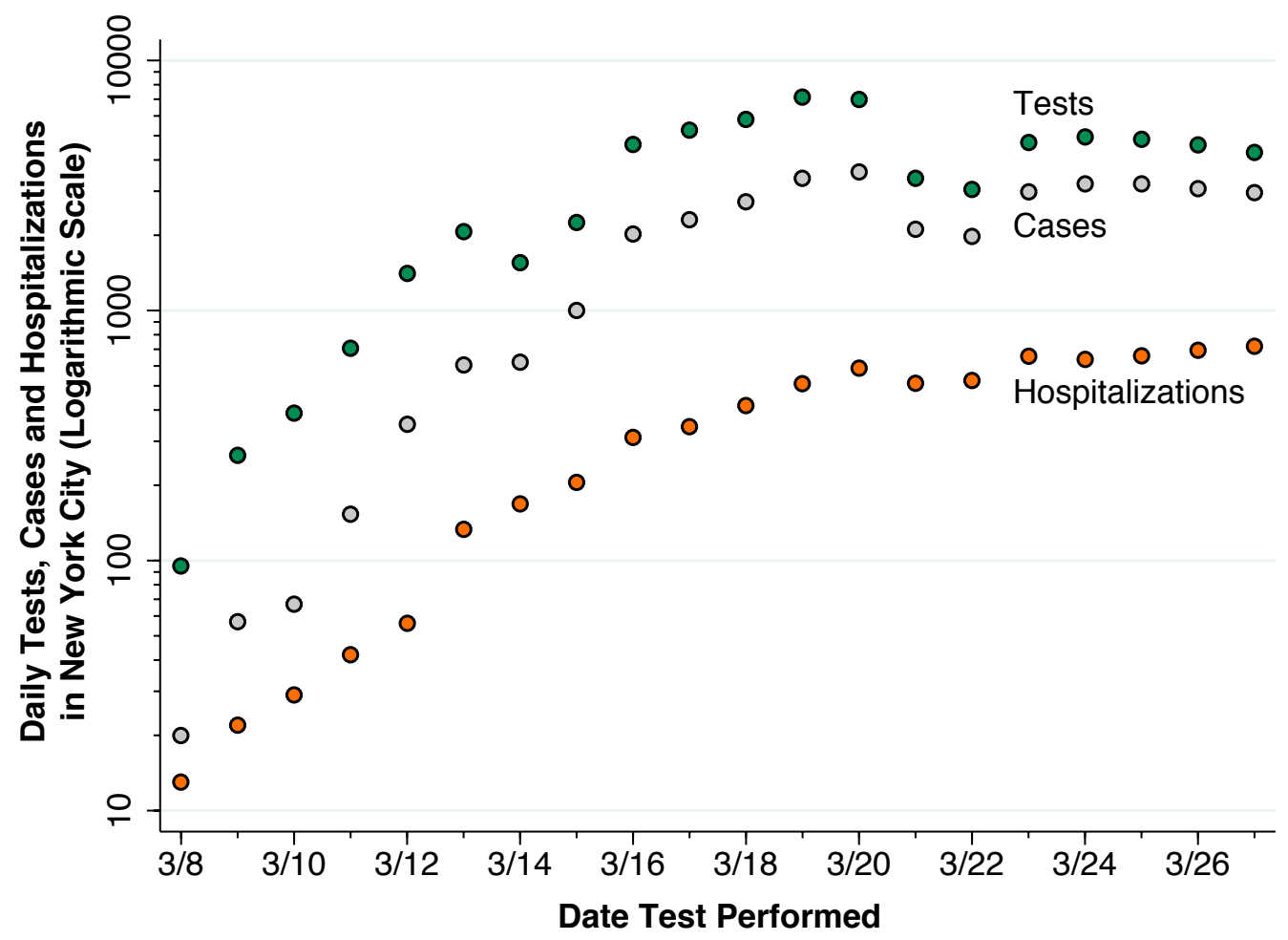

Figure 3. Daily Counts of Tests Performed, New Cases Identified, and New Cases Hospitalized in New York City (Logarithmic Scale), Based on Date Test Performed. Observations cover tests performed from March 8-27, 2020.

The horizontal axis in Figure 1 for New York City and Figure 2 for Los Angeles County measured the date each test result was received. That allowed us to compare the trends in the two regions. The further advantage of reporting by date received is that the reporting agency doesn't have to keep updating its past case counts. The horizontal axis in Figure 3, by contrast, measures 
the date each test was performed. Unfortunately, displaying the data in that manner runs into a messy statistical problem (Harris 1990b). The reporting agency has to keep updating past counts every time a new case is received. What's worse, the most recent data points are invalid because all the cases haven't yet come in. Accordingly, we have omitted the data points after March 27, which are subject to revision.

The light gray-colored points in Figure 3 confirm the pattern for New York City seen in Figure 1. There is a rapid initial phase followed by a leveling off of counts of new cases. The green-colored points confirm that the number of tests performed was considerably greater than the number of positive test results. While there is a temporary dip in the number of tests performed during March 21-22, right after the Department of Public Health ordered providers to restrict testing, the number of tests performed daily quickly rebounded. Most important, the number of hospitalized cases has followed the same basic pattern - an initial takeoff followed by a plateau.

Table 1 further interprets the data in Figure 3, aggregating the testing dates into five-day intervals. The rightmost column shows that healthcare providers in New York City have not been testing just hospitalized COVID-19 patients. If they were, new COVID-19 hospitalizations as a fraction of all newly reported COVID-19 cases would be a whole lot higher than 22 percent. What's more, the percentage would have increased markedly during the more recent interval. In fact, the ratio of hospitalized COVID-19 cases to all reported COVID-19 cases in New York City is entirely compatible with data reported by the Centers for Disease Control for the entire U.S. (CDC COVID-19 Response Team 2020), as well as data from Wuhan, China (Wang et al. 2020).

Table 1. Number of Positive Tests, Ratio of Tests Performed to Positive Tests, and Percentage of Positive Tests Linked to Hospitalization for 5-Day Testing Intervals, New York City.

\begin{tabular}{|c|c|c|c|}
\hline Dates Performed & $\begin{array}{c}\text { Number of Positive } \\
\text { COVID-19 Tests }\end{array}$ & $\begin{array}{c}\text { Ratio of Tests } \\
\text { Performed to } \\
\text { Positive Tests }\end{array}$ & $\begin{array}{c}\text { Percentage of } \\
\text { Positive Tests Linked } \\
\text { to Hospitalization }\end{array}$ \\
\hline $3 / 9-3 / 13 / 20$ & 1,234 & 3.9 & $22.9 \%$ \\
\hline $3 / 14-3 / 18 / 20$ & 8,670 & 2.2 & $16.7 \%$ \\
\hline $3 / 19 / 20-3 / 23 / 20$ & 14,044 & 1.8 & $19.9 \%$ \\
\hline $3 / 24 / 20-3 / 28 / 20$ & 14,461 & 1.5 & $22.2 \%$ \\
\hline
\end{tabular}


As to the assertion that the supply of tests has been severely limited, the available evidence is that the state of New York has been way ahead of other states in coronavirus testing, having screened 32,400 people by March 20 alone (Klersz 2020). By March 30, New York City alone had tested over 79,000 individuals - more than twice as many tests as confirmed COVID19 infections - while New York state had tested over 186,000 (Choi et al. 2020). Table 1 shows that even during the last five-day interval, the number of tests was still 50-percent greater than the number of infections.

\section{Alternative Explanation \#2. We're Missing All the Asymptomatic Cases.}

It is widely acknowledged that many individuals infected by coronavirus have too few symptoms to seek medical attention, yet they can still serve as carriers, transmitting the infection to others. That observation alone would lead us to suspect that the counts of newly diagnosed cases in Figure 1 substantially understate the true daily incidence - a statistic we could reliably observe only if we had universal mandatory testing. However, there is no clear reason to believe that the extent of understatement has changed significantly since the one-month takeoff period covered in Figure 1. So long as the ratio of undetected asymptomatic cases to detected symptomatic cases has remained constant, we would still observe the flattening in the logarithmic plot in the figure even if we had complete data on all cases. Put differently, the underreporting bias affects the magnitude but not the shape of the incidence curve.

Perhaps the best data on the ratio of asymptomatic to symptomatic cases comes from the Diamond Princess cruise ship, a closed population in which everyone's COVID-19 infection status was ascertained (Russell et al. 2020). Out of a total of 3,711 tested Diamond Princess passengers of all ages, 619 (or 13\%) were infected. Among the 619 infected passengers, 301 (or $49 \%$ ) were asymptomatic. That gives us the rule of thumb that, for every symptomatic infected person seeking medical attention, there's another asymptomatic infected person who didn't. In the forecasts of the London modeling group, these asymptomatic infected people are an important force driving the epidemic (Li, Pei, et al. 2020). They may be less infectious than their symptomatic counterparts, but there are so many of them.

It is arguable that the ratio of undocumented asymptomatic infections to documented symptomatic infections depends on the age distribution of the population. But the Diamond Princess data do not show a marked difference in this ratio by age. Among those infected passengers under age 70, a total of 53 percent had few or no symptoms. Among those infected 
passengers age 70 or more, a total of 43 percent were asymptomatic, not much different from the asymptomatic proportion in the younger group (Russell et al. 2020).

In short, there is no obvious biological or economic reason why the proportion of undocumented asymptomatic cases has changed enough to explain the marked flattening of the trend in documented cases seen in Figure 1.

\section{Alternative Explanation \#3. We're Missing the False Negatives.}

Tests for coronavirus infection appear to be highly specific. That is, if the test is positive, the clinician can be confident that the patient is in fact infected. But not all tests are sensitive. Depending on the sample taken and the timing of the test, the result could be a false negative. The available data from the Wuhan outbreak indicate that tests based on a sample of the patient's sputum taken during the first two weeks of infection are pretty sensitive, with a false negative rate in the range of $11.1-25.6 \%$. But tests based on nasal swabs, which are the most common thus far in the U.S., are considerably less sensitive, with a false negative rate in the range of 26.7-46.4\% (Yang et al. 2020). That means routine nasal swab testing could be missing a lot of coronavirus infections.

As in our consideration of Alternative Explanation \#2, the issue here is not how many genuine cases of COVID-19 have been missed as a result of insensitive testing, but whether the percentage of false positives has changed significantly during the month of March 2020 in New York City. The evidence from the Wuhan outbreak is that samples taken after the first two weeks of infection had higher false positive rates in the range of 38.9-57.1\%. However, we have no data to indicate that coronavirus testing has been significantly delayed in recent days.

\section{Alternative Explanation \#4. We Need to Break Down the Data by Borough.}

Figure 1 aggregated the data from all five boroughs of New York City. The figure did not address the possibility that in one or more boroughs, the epidemic may be continuing to grow at an exponential rate comparable to the initial takeoff. The concern is heightened by reports that such hospitals as Elmhurst in Queens and Columbia Presbyterian in The Bronx have been flooded with COVID-19 patients (Hopkins 2020). What's more, the Department of Public Health has released a heat map of the city showing that in certain neighborhoods of the Bronx, Queens and Brooklyn, the proportion of positive tests has exceeded 56 percent (New York Department of Health and Mental Hygiene 2020e). Overall, the prevalence of positive tests was 561 per 
100,000 population in Queens and 530 per 100,000 in The Bronx, compared to 351 per 100,000 in Manhattan (New York Department of Health and Mental Hygiene 2020c).

Figure 4 plots the counts of newly reported cases from March 21 onward for the four principal boroughs of the city. While the daily counts are noisy, the burden of disease is greater in Queens and Brooklyn. Still, all of the estimated doubling times are substantially longer than that of the initial takeoff of 1.3 days seen in Figure 1. The data points for Manhattan suggest that incidence in this borough may in fact have reached a flat peak and leveled off.

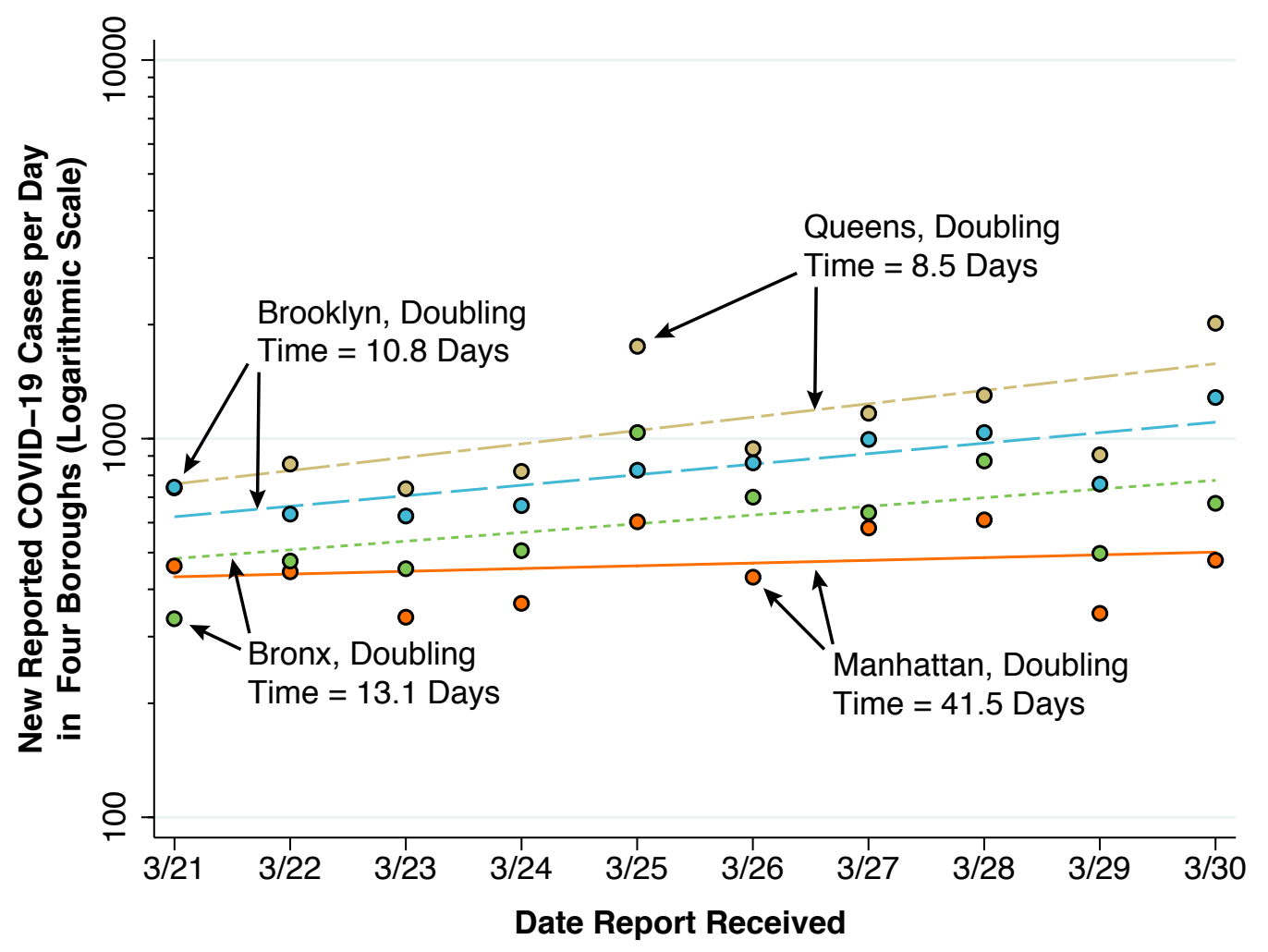

Figure 4. New Reported COVID-19 Cases per Day in Four New York City Boroughs (Logarithmic Scale), Based on Date Reported. Best-fit trend lines (Poisson regression) and estimated doubling times are based upon observations from 3/21/2020 onward. The data and regression line for each borough are distinguished by color: lilac for Queens, cyan for Brooklyn, lime for the Bronx, and orange for Manhattan.

\section{Alternative Explanation \#5. The Leaky City}

New York City has not been under complete lock down. People enter and exit. While the streets of Manhattan area deserted, it's not necessarily because all Manhattanites are staying home, but because they have fled. While there is substantial anecdotal evidence supporting this explanation (Leavenworth 2020, Hampton 2020, Scicchitano 2020), we have been unable to find 
any quantitative estimates of the magnitude of the exodus. If those who have fled the city were less likely to be infected, the leak would have raised rather than lowered the observed incidence.

For the sake of argument, let's assume that those who fled the city were just as likely to be infected. For this theory alone to explain the apparent flattening of the curve in Figure 1, well over 90 percent of the population of New York City would have to have joined the coronavirus diaspora.

\section{Alternative Explanation \#6. The Rapid Initial Upswing Is Unrealistic.}

Is the initial doubling time of only 1.3 days in New York City unrealistic? As noted in Appendix 2, we estimated the slope of the initial takeoff to be 0.53 . Using the formula in Appendix 1, we computed a doubling time of $(\log 2) / 0.53=1.3$ days. In general, the longer an infected person remains contagious, the more rapidly the epidemic grows. Data from Wuhan provide a reasonable estimate of the average duration of infectivity of 6.5 days (Ferguson et al. 2020). Using another formula in Appendix 1, we estimate that the basic reproductive number $\boldsymbol{R}_{0}$ in New York City at the start of its epidemic was $6.5 \times 0.53$, which comes to about 3.4. If we perform the same exercise for the Los Angeles County data in Figure 2, we get a basic reproductive number of $\boldsymbol{R}_{0}=1.8$. The estimated reproductive number for Wuhan, which has a population density of about 11,400 persons per square mile, has been estimated to be about $\boldsymbol{R}_{0}=$ 2.2 to 2.4 (Li, Guan, et al. 2020, Riou and Althaus 2020). Accordingly, the slope of the initial takeoff for New York City shown in Figure 1 is hardly unrealistic, and in fact is entirely consistent with its extraordinarily high population density in comparison even to Wuhan.

\section{Alternative Explanation \#7. Heterogeneous Mixing or Super-Spreaders}

One interpretation of these findings is that the apparent flattening of the incidence curve in Figure 1 is a result of extreme heterogeneity in the infectivity of the New York City population, with a small proportion of the total population - on the order of 5,000 individuals subject to the super-spread of the virus. While there are anecdotal reports of such super-spread in the New York City area (Williamson and Hussey 2020), we haven't found clear evidence of a major source comparable to the 77 COVID-19 cases reportedly emanating from the late February Biogen meeting in Boston (Marcelo and O’Brien 2020). 


\section{Alternative Explanation \#8. Where Are the Supporting Ancillary Data?}

If the leveling off observed in Figure 1 is real, then we would expect to see the same pattern in supporting data. We've already looked at hospitalizations in Figure 3. The next likely candidate would be the daily numbers of deaths in New York City. This source of data has its own problems, not the least of which is it comes from the same agency as the case and hospitalization data, and may thus be subject to common biases. What's more, the observed patterns may be complicated by significant further time delays. By one estimate, it takes an average of 16 days from the onset of symptoms until a patient dies of complications (Muzimoto and Chowell 2020). With an incubation period from initial infection to onset of symptoms averages 5 days (Linton et al. 2020, Li, Guan, et al. 2020), the combined time from initial infection to death will average about three weeks.
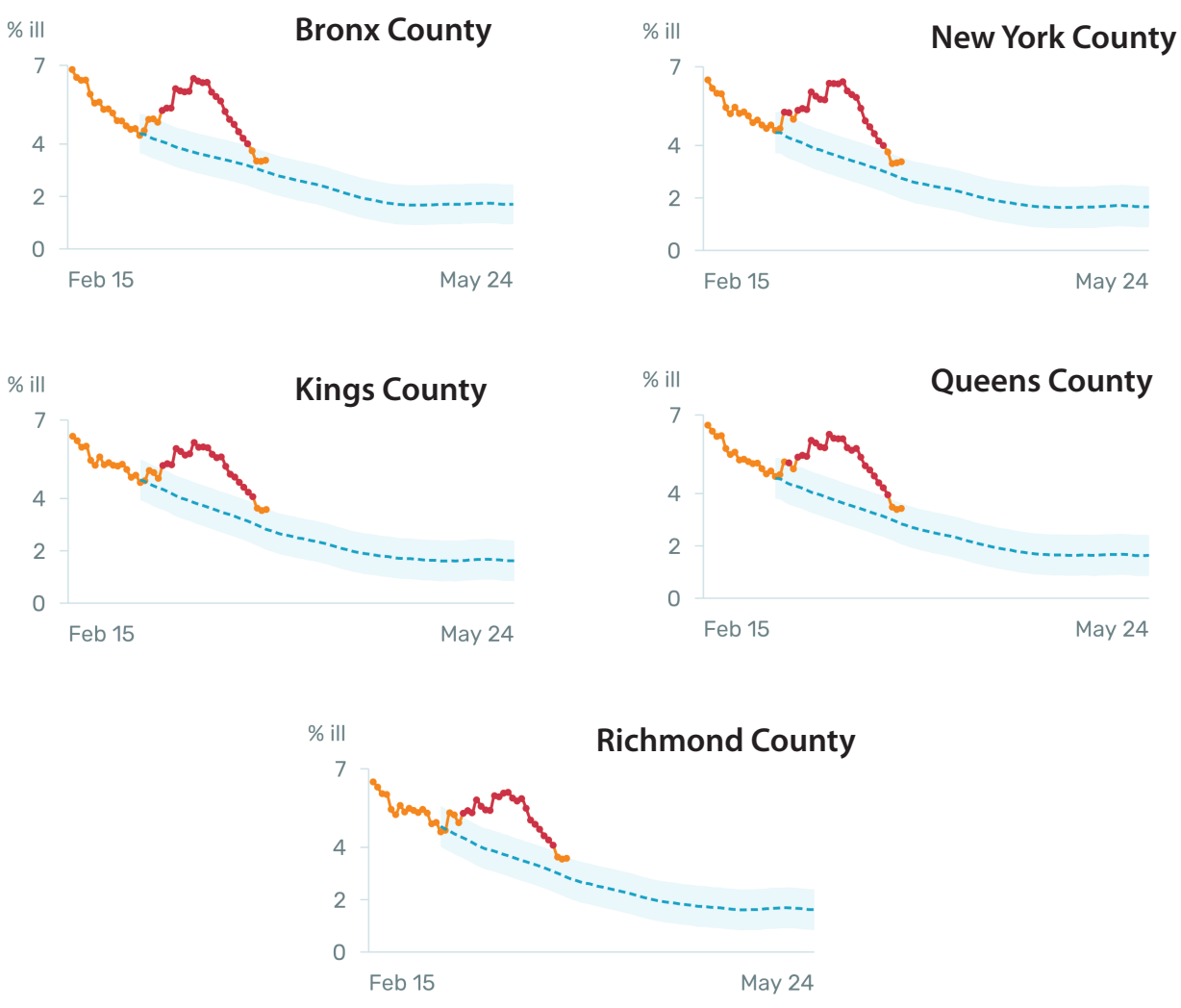

Figure 5. Plots of the Percentage with Influenza-Like Illness Reported by Kinsa, Inc. in Five Counties in the New York City Area, February 15 - March 30, 2020. 
We therefore looked for an ancillary data source that is timely and independent of the New York City Department of Health. To that end, Figure 5 above shows the trends in influenzalike illness monitored at the U.S. Health Weather Map, a compilation based on recordings of Kinsa, Inc.'s network of smart thermometers, produced in collaboration with Benjamin Dalziel at Oregon State University (Kinsa 2020, Dalziel 2020). The figure displays the estimated percentages with febrile illness in each of the five counties comprising the New York City area during February 15 - March 30, 2020. The dashed blue curve, along with the surrounding light blue confidence range, shows the typical percentage with influenza-like illness. The orange data points show the observed percentages in each county, and the red data points indicate where the observed percentages fall above the confidence range.

In each county, the percentages with influenza-like illness began to rise at the beginning of March, just when the influenza season was subsiding. By the middle of March, however, the prevalence of influenza-like illness has reversed itself and begins to decline toward the expected baseline. These patterns, derived from data wholly independent from reports of the New York Department of Health, support our findings in Figure 1.

\section{If the Flattening of the Curve is Real, How Did It Happen?}

None of the foregoing alternative explanations is mutually exclusive. In fact, it is entirely possible that some of these alternative explanations are at least partially valid and that the epidemic curve in New York City is flattening. Based on the evidence reviewed here, however, it is difficult to dismiss out of hand the conclusion that the incidence of new coronavirus infections has indeed leveled off.

This does not mean that the epidemic has reached its peak in New York City. That will happen when the incidence of new infections clearly drops. Still, the leveling off of the incidence curve is a key early indicator that something favorable is happening. The question for economists, public health practitioners and policy makers is: what's making the curve flatten?

There is certainly evidence that social distancing measures reduce transmission and flatten the curve. That includes an important study comparing the containment policies of different cities during the 1918 H1N1 influenza pandemic (Markel et al. 2007). The recent experiences of Singapore, Hong Kong and Taiwan during the current COVID-19 pandemic, where social distancing measures were combined with aggressive case tracking, add credence to the conclusion that public policies can be effective (Branswell 2020). In view of the five-day 
incubation period from infection to symptoms, the observed decline starting on March 21 in Figure 1 would thus be consistent with Mayor de Blasio's order limiting gatherings and closing gyms and other places of congregation. It would be reinforced by subsequent orders that all New Yorkers except workers in essential businesses must stay at home.

There is, however, another strand in the economics literature suggesting that people voluntarily engage in avoidance behaviors once they fully perceive the risks of contagion. In a study of the time path of the H1N1 Swine Flu epidemic of 2009 in the U.S. - when there were no quarantines or lock downs - economists could see no other explanation for their finding that the incidence of new cases fell well below the exponential path predicted from epidemiologists' classical models (Yoo, Kasajima, and Bhattacharya 2010). Similarly, the incidence of HIV reached a peak in 1983 well before the advent of the first antiviral medication in 1986 (Harris 1990a, Harris 1990b) and about a year before San Francisco's Director of Public Health ordered the closure of fourteen bathhouses in October 1984. In the present context, ridership on New York City subways was already down 19 percent by March 12 and 60 percent by March 16 (Metropolitan Transportation Authority 2020). In all of these cases, the critical ingredient in the public policy mix may have been the successful communication of consistent, clear, accurate and timely information to millions of individuals, who responded by taking action without government coercion. Put bluntly, what flattened the curve was no more than the naked truth.

Still, the two approaches to flattening the epidemic curve are hardly incompatible, or even distinct. The promulgation of strong social distancing measures, in fact, may be the best way to reinforce the credibility of the risk message. The mayor's and governor's orders may have been so impactful that they needed little or no formal enforcement. With the wide publicity that many New York City hospitals were already triaging intensive care, one also wonders how many people would actually go back to their regular daily activities if the restrictions on social distancing were abruptly - and foolishly - withdrawn tomorrow.

There is a third related behavioral theory that people are most motivated to take action to reduce risk when they personally come in contact with other affected individuals. For example, having a family member with a diagnosis of cancer enhances an individual's intention to quit smoking (Patterson et al. 2010). These considerations might help explain why we have observed a slowing of COVID-19 incidence in New York City, but not so far in Los Angeles County. In New York City to date, about 1 out of every 226 residents is known so far to have contracted 
COVID-19. The probability of knowing at least one coronavirus-infected person in a social circle of 150 persons would be one-half. In Los Angeles, by contrast, only about 1 out of 4,100 residents is so far known to have come down with the virus. The corresponding probability of knowing at least one infected person in a comparable size social circle of 150 would be only about 3.4 percent.

As this working paper goes to press, the New York Times is running a piece entitled N.Y.C. 's 911 System Is Overwhelmed. 'I'm Terrified,' a Paramedic Says, with the subtitle, "With coronavirus cases mounting, emergency workers are making life-or-death decisions about who goes to a hospital, and who is left behind." (Watkins 2020) It would seem to offer little solace that the number of newly reported coronavirus victims in New York City appears to have leveled off at about 3,200 per day, of whom about 700 need to be hospitalized. And it certainly won't make anyone feel any better that, if the social distancing policies promulgated by the city and state governments had been put in place just one week earlier, we might now be fretting about hundreds rather than thousands of newly reported infections daily.

Still, the message of the present analysis is clear. Hang in there, hold tight, and see what happens.

\section{Appendix 1. SIR Epidemic Model}

The classic Kermack-McKendrick model (Kermack and McKendrick 1991), first published in 1927 and now called the SIR model, continues to serve as the basis for projections of the path of epidemics, including the COVID-19 epidemic. According to the basic version of the model, the time course of an epidemic can be described by three coupled differential equations. Let $S(t)$ denote the number of susceptible individuals, $I(t)$ denote the number of infective individuals, and $R(t)$ denote the number of resistant individuals at time $t \geq 0$. All individuals in the population are in one of these three states. In the basic version of the model, which we adopt here, the population is assumed closed, so that $S(t)+I(t)+R(t)=N$, where $N$ is a time-independent constant. That is, $\dot{S}+\dot{I}+\dot{R}=0$, where we have used the notation $\dot{S}=\frac{d S(t)}{d t}$ for the first derivative. 
The rate of new infections per unit time is assumed to be proportional to the number of interactions between susceptible and infective individuals, that is, $-\dot{S}=\alpha(S / N) I$, where $\alpha>0$ is a constant. Once an individual is infected, he is infective and remains infective until he becomes resistant, either through recovery or death. Infective individuals are assumed to become resistant at a constant proportional rate, that is, $\dot{R}=\beta I$, where $\beta>0$ is also a constant. Since our population is closed, we have $\dot{I}=-\dot{S}-\dot{R}=\alpha(S / N) I-\beta I$. For notational convenience, without adding any additional states to the model, we write the number of individuals ever infected as $C=I+R=N-S$.

Our focus here is on an epidemic where everyone starts out naïve to the infectious agent, that is, where $R(0)=0$. If the initial number of infectives $I(0)$ is very small, then the initial susceptible population $S(0)=N-I(0)$ is approximately constant and equal to $N$, and thus $S / N$ is approximately equal to 1. Accordingly, during the initial takeoff phase of such an epidemic, the number of newly infected individuals per unit time, will be approximately $\dot{C}=\alpha I$. Since $R$ will initially be small, we have the further approximation $\dot{C}=\alpha C$. Put differently, during the takeoff phase of an epidemic where no one is initially resistant, the cumulative number of individuals infected will approximately follow the exponential model $C(t)=I(0) \exp (\alpha t)$. The number of newly infected individuals per unit time will likewise follow the exponential model $\dot{C}(t)=I(0) \alpha \exp (\alpha t)$. Equivalently, $\log \dot{C}(t)=\log (I(0))+\log (\alpha)+\alpha t$.

The key model parameter $\alpha$ captures the rate of contact among susceptible and infective individuals as well as the likelihood that such a contact results on average in a new infection. Social distancing would thus be expected to reduce $\alpha$. To assess whether social distancing has in fact reduced $\alpha$, we can plot the number of new infections on a logarithmic scale versus the time elapsed since the start of the epidemic. Once we have an estimate of the parameter $\alpha$, the doubling time of new infections is given by $(\log 2) / \alpha$.

The differential equation for the number of infectives $\dot{I}=-\dot{S}-\dot{R}=\alpha(S / N) I-\beta I$ can be rewritten as $\dot{I}=(\alpha(S / N)-\beta) I$. The sign of the growth rate $\dot{I}$ in the number of infectives thus depends on the sign of $\alpha(S / N)-\beta$. Equivalently, the epidemic is increasing when the 
reproductive number $\boldsymbol{R}=\frac{\alpha S}{\beta N}$ exceeds 1 , while it is decreasing when $\boldsymbol{R}<1$. At the start of an epidemic where nearly everyone is susceptible and $S$ is approximately equal to $N$, the basic reproductive number is therefore $\boldsymbol{R}_{0}=\alpha / \beta$. This represents the average number of new infections resulting from a given infection during the initial takeoff of the epidemic. The average duration of infectivity appears to be approximately 6.5 days (Ferguson et al. 2020), though data from more severely ill patients suggest an infective period up to 10 days (Wölfel et al. 2020). For a 6.5-day infective period among all patients, we would have $\beta=1 / 6.5$. Accordingly, once we have an estimate of $\alpha$ from the logarithmic growth rate of the initial takeoff phase of the epidemic, we approximate the basic reproductive number as $\boldsymbol{R}_{0}=6.5 \alpha$.

\section{Appendix 2. Poisson Regression Estimates}

The estimated trend lines for the takeoff phase of the epidemic in Figures 1 and 2 were estimated by Poisson regression, an appropriate statistical method when the dependent variable involves count data, as was the case here. Here are the results for the two figures.

\begin{tabular}{|l|c|c|c|}
\hline \multicolumn{1}{|c|}{ Data Set } & $\begin{array}{c}\text { Number of } \\
\text { Observations }\end{array}$ & $\begin{array}{c}\text { Estimated } \\
\text { Slope }\end{array}$ & $\begin{array}{c}\text { 95\% Confidence } \\
\text { Interval }\end{array}$ \\
\hline New York City (Figure 1) & 20 & 0.527 & $0.513-0.541$ \\
\hline Los Angeles County (Figure 2) & 19 & 0.266 & $0.232-0.300$ \\
\hline
\end{tabular}

\section{References}

Branswell, H. 2020. "Understanding what works: How some countries are beating back the coronavirus." STAT News (March 20, 2020).

CDC COVID-19 Response Team. 2020. "Severe Outcomes Among Patients with Coronavirus

Disease 2019 (COVID-19) — United States, February 12-March 16, 2020." MMWR 69 (12):343-346.

Choi, A., Y. Gonen, J. Velasquez, and W. Welch. 2020. Coronavirus in New York City: Tracking the spread of the pandemic. https://projects.thecity.nyc/2020_03_covid-19-tracker/: COVID Trackint Project, March 27, 2020. 
County of Los Angeles Public Health. 2020. Novel Coronavirus in Los Angeles County. http://www.publichealth.lacounty.gov/media/Coronavirus/: (Regularly updated).

Dalziel, B. 2020. Web portal. https://ib.oregonstate.edu/faculty/benjamda/Benjamin-Dalziel: Oregon State University Department of Integrative Biology.

Ferguson, N.M., D. Laydon, G. Nedjati-Gilani, and et al. 2020. Impact of non-pharmaceutical interventions (NPIs) to reduce COVID-19 mortality and healthcare demand. https://www.imperial.ac.uk/media/imperial-college/medicine/sph/ide/gidafellowships/Imperial-College-COVID19-NPI-modelling-16-03-2020.pdf: March 16, 2020.

Hampton, Daniel. 2020. "NYC Exodus Infecting LI; 2-Week Self-Quarantine Recommended." Pathc (Levittown NY) (March 24, 2020).

Harris, J. E. 1990a. "Improved short-term survival of AIDS patients initially diagnosed with Pneumocystis carinii pneumonia, 1984 through 1987." JAMA 263 (3):397-401.

Harris, J.E. 1990b. "Reporting delays and the indicence of AIDS." Journal of the American Statistical Association 85 (412):915-924.

Harris, S., and F.S. Sellers. 2020. "Inside a major New York City hospital system battling coronavirus." Washinton Post (March 28, 2020).

Hopkins, C. 2020. "Covid-19 patients are flooding New York hospitals, and the peak may be 3 weeks away." Vox (March 27, 2020).

Kermack, W.O., and A.G. McKendrick. 1991. "A contribution to the mathematical theory of epidemics - I." Bulletin of Mathematical Biology (reprinted) 53 (1-2):33-55.

Kinsa, Inc. 2020. U.S. Health Weather Map. https://healthweather.us/ (as of 3/30/2020).

Klersz, Andy. 2020. "https://www.businessinsider.com/new-york-more-coronavirus-testing-thanthe-cdc-any-state-2020-3." Business Insider (March 20).

Leavenworth, Jesse. 2020. "Escape from New York: City dwellers fleeing coronavirus are flocking to Connecticut." Hartford Courant (March 26, 2020).

Li, Q., X. Guan, P. Wu, and et al. 2020. "Early Transmission Dynamics in Wuhan, China, of Novel Coronavirus-Infected Pneumonia." N Engl J Med 382 (13):1199-1207. doi: 10.1056/NEJMoa2001316. 
Li, R., S. Pei, B. Chen, Y. Song, T. Zhang, W. Yang, and J. Shaman. 2020. "Substantial undocumented infection facilitates the rapid dissemination of novel coronavirus (SARSCoV2)." Science. doi: 10.1126/science.abb3221.

Linton, N.M., T. Kobayashi, Y. Yang, and et al. 2020. Epidemiologicalcharacteristics of novel coronavirus infection: A statistical analysis of publicly available case data. https://www.medrxiv.org/content/medrxiv/early/2020/01/28/2020.01.26.20018754.full.pd f: February 18, 2020.

Marcelo, P., and M. O’Brien. 2020. "Cluster of Coronavirus Cases Tied to U.S. Biotech Meeting." Time (March 11, 2020).

Markel, H., H. B. Lipman, J. A. Navarro, A. Sloan, J. R. Michalsen, A. M. Stern, and M. S. Cetron. 2007. "Nonpharmaceutical interventions implemented by US cities during the 1918-1919 influenza pandemic." JAMA 298 (6):644-54. doi: 10.1001/jama.298.6.644.

Metropolitan Transportation Authority. 2020. MTA Annual Disclosure Statement Supplement. http:/web.mta.info/mta/investor/pdf/2020/2019CoronavirusUpdateSupp031820.pdf: March 18, 2020.

Muzimoto, K., and G. Chowell. 2020. "Estimating Risk for Death from 2019 Novel Coronavirus Disease, China, January-February 2020." Emerging Infectious Diseases 26 (6 (June)):https://wwwnc.cdc.gov/eid/article/26/6/20-0233-techapp1.pdf. doi: 10.3201/eid2606.200233.

New York Department of Health and Mental Hygiene. 2020a. 2020 Advisory \#8: COVID-19 Update for New York City. https:/www1.nyc.gov/assets/doh/downloads/pdf/han/advisory/2020/covid-1903202020.pdf: March 20, 2020.

New York Department of Health and Mental Hygiene. 2020b. Coronavirus Disease 2019 (COVID-19)

. https://www1.nyc.gov/site/doh/covid/covid-19-main.page: (Regularly updated).

New York Department of Health and Mental Hygiene. 2020c. COVID-19: Data. https://www1.nyc.gov/site/doh/covid/covid-19-data.page: March 31, 2020.

New York Department of Health and Mental Hygiene. 2020d. nyc health/coronavirus-data. https://github.com/nychealth/coronavirus-data: testing.csv, March 31, 2020. 
New York Department of Health and Mental Hygiene. 2020e. Percent of patients testing positive for COVID-19 by United Hospital Fund Neighborhood in New York City as of March 29, 2020. https://www1.nyc.gov/assets/doh/downloads/pdf/imm/covid-19-data-map.pdf: March 30, 2020.

Patterson, F., E. P. Wileyto, J. Segal, J. Kurz, K. Glanz, and A. Hanlon. 2010. "Intention to quit smoking: role of personal and family member cancer diagnosis." Health Educ Res 25 (5):792-802. doi: 10.1093/her/cyq033.

Riou, J., and C. L. Althaus. 2020. "Pattern of early human-to-human transmission of Wuhan 2019 novel coronavirus (2019-nCoV), December 2019 to January 2020." Euro Surveill 25 (4). doi: 10.2807/1560-7917.ES.2020.25.4.2000058.

Russell, T.W., J. Hellewell, C.I. Jarvis, and et al. 2020. Estimating the infection and case fatality ratio for COVID-19 using age-adjusted data from the outbreak on the Diamond Princess cruise ship. https://www.medrxiv.org/content/10.1101/2020.03.05.20031773v2: Centre for the Mathematical Modelling of Infectious Diseases, Department of Infectious Disease Epidemiology, London School of Hygiene and Tropical Medicine, London, March 5, 2020.

Scicchitano, Paul. 2020. "FL Orders NY, NJ, CT Travelers To Self-Quarantine For Coronavirus." Patch (Miami.FL) (March 23, 2020).

Wang, D., B. Hu, C. Hu, F. Zhu, X. Liu, J. Zhang, B. Wang, H. Xiang, Z. Cheng, Y. Xiong, Y. Zhao, Y. Li, X. Wang, and Z. Peng. 2020. "Clinical Characteristics of 138 Hospitalized Patients With 2019 Novel Coronavirus-Infected Pneumonia in Wuhan, China." JAMA. doi: 10.1001/jama.2020.1585.

Watkins, A. 2020. "N.Y.C.'s 911 System Is Overwhelmed. 'I’m Terrified,' a Paramedic Says." New York Times (March 28, 2020).

Williamson, E., and C. Hussey. 2020. "Party Zero: How a Soirée in Connecticut Became a 'Super Spreader'." New York Times (March 23, 2020).

Wölfel, R., V.M. Corman, W. Guggemos, and et al. 2020. Virological assessment of hospitalized cases of coronavirus disease 2019 https://www.medrxiv.org/content/10.1101/2020.03.05.20030502v1.full.pdf: March 5, 2020. 
Yang, Y., M. Yang, C. Shen, and et al. 2020. Evaluating the accuracy of different respiratory specimens in the laboratory diagnosis and monitoring the viral shedding of 2019-nCoV infections. https://www.medrxiv.org/content/10.1101/2020.02.11.20021493v2.full.pdf: February 17, 2020.

Yoo, B-K., M. Kasajima, and J. Bhattacharya. 2010. Public Avoidance and the Epidemiology of Novel H1N1 Influenza. https://www.nber.org/papers/w15752.pdf: National Bureau of Economic Research Working Paper 15752, February 2010. 